\title{
Avaliação do grau de deterioração da estrutura da ponte localizada no campus da Universidade Federal de Santa Maria no RS
}

\author{
G. D. Brackmann ${ }^{1 *}$, C. de Vargas ${ }^{1}$, E. M. de $\operatorname{Carli}^{1}$, G. F. dos $\operatorname{Santos}^{1}$, R. C. A. de Lima ${ }^{2}$, A. S. \\ de Vargas $^{2}$ \\ *Autor de Contato: gabrielabrackmann@hotmail.com

\footnotetext{
${ }^{1}$ Estudante de Mestrado, Programa de Pós-Graduação em Engenharia Civil, Universidade Federal de Santa Maria, Santa Maria, Brasil

${ }^{2}$ Professor, Programa de Pós-Graduação em Engenharia Civil, Universidade Federal de Santa Maria, Santa Maria,
} Brasil

\section{RESUMO}

Neste trabalho foi realizada a avaliação do grau de deterioração da estrutura $\left(\mathrm{G}_{\mathrm{d}}\right)$ da ponte localizada dentro do campus da Universidade Federal de Santa Maria, utilizando a metodologia GDE/UnB. A partir da inspeção visual da estrutura, avaliou-se a ocorrência de manifestações patológicas nos elementos estruturais e o seu grau de intensidade, possibilitando a determinação do $\mathrm{G}_{\mathrm{d}}$ igual a 75,90 , equivalente a um nível de deterioração alto, para o qual recomenda-se a realização de uma inspeção especializada e o planejamento de uma intervenção no prazo de 1 ano. A metodologia GDE/UnB mostrou-se eficaz para definir a ordem de prioridade de intervenções na estrutura, assim, a partir dos resultados obtidos com o método, foi proposta uma lista de intervenções necessárias na ponte.

Palavras-chave: manifestações patológicas; inspeção; pontes; grau de deterioração. 


\begin{abstract}
In this work, the degree of deterioration of the structure $\left(\mathrm{G}_{\mathrm{d}}\right)$ of the bridge located within the campus of the Federal University of Santa Maria was carried out, using the GDE/UnB methodology. The occurrence of pathological manifestations in the structural elements and their degree of intensity were evaluated during the visual inspection of the bridge, enabling the determination of $\mathrm{G}_{\mathrm{d}}$ equal to 75.90 , which is equivalent to a high level of deterioration, being recommended the performance of a specialized inspection and the planning of an intervention within 1 year. The GDE/UnB methodology proved to be effective in defining the order of priority of interventions in the structure, thus, based on the results obtained, a list of necessary interventions on the bridge was proposed.
\end{abstract}

Keywords: pathological manifestations; inspection; bridges; degree of deterioration.

\title{
RESUMEN
}

En este trabajo se realizó el grado de deterioro de la estructura $\left(\mathrm{G}_{\mathrm{d}}\right)$ del puente ubicado dentro del campus de la Universidad Federal de Santa Maria, utilizando la metodología GDE/UnB. A partir de la inspección visual de la estructura se evaluó la ocurrencia de manifestaciones patológicas en los elementos estructurales y su grado de intensidad, posibilitando la determinación de $\mathrm{G}_{\mathrm{d}}$ igual a 75,90 , equivalente a un alto nivel de deterioro, para lo cual recomendamos si la realización de una inspección especializada y la planificación de una intervención en el plazo de 1 año. La metodología GDE/UnB demostró ser efectiva en la definición del orden de prioridad de las intervenciones en la estructura, por lo que a partir de los resultados obtenidos con el método se propuso una lista de intervenciones necesarias en el puente.

Palabras clave: manifestaciones patológicas; inspección; puentes; grado de deterioro.

\section{INTRODUÇÃO}

De forma geral, as manifestações patológicas são evolutivas e tendem a se agravar com o passar do tempo se não ocorrerem intervenções para manutenção e/ou recuperação, quando necessário. Além disso, segundo Helene (2003), o custo associado a uma intervenção possui um aumento exponencial em função do tempo, destacando a importância das vistorias e inspeções nas estruturas para o acompanhamento do estado de conservação e planejamento das intervenções. As inspeções têm o objetivo de identificar e acompanhar o desenvolvimento de anomalias e patologias nos elementos estruturais, resultando em manutenções menos onerosas e contribuindo para a manutenção da segurança estrutural e das condições de serviço.

A Universidade Federal de Santa Maria (UFSM) foi a primeira universidade federal criada fora de uma capital brasileira e representou um marco importante no processo de interiorização do ensino universitário público no Brasil. A UFSM foi idealizada e fundada pelo Prof. Dr. José Mariano da Rocha Filho em 1960, tornando o Rio Grande do Sul o primeiro estado da federação a contar com duas universidades federais. Parte integrante da infraestrutura da cidade universitária é a Ponte Marechal Cândido Mariano Rondon (Figura 1), que faz a ligação entre os dois extremos do campus. A Obra de Arte Especial (OAE) foi construída em 1971, possui 108 metros de extensão e leva o título de "ponte" pois o projeto urbanístico original da universidade previa a construção de um lago artificial sob ela; no entanto, essa obra nunca ocorreu. 
Após 50 anos da construção da OAE, é notável a mudança ocorrida nas características e no volume de automóveis que passam pela estrutura diariamente, sendo, portanto, de extrema importância para a logística de tráfego dentro do campus garantir a manutenção do desempenho em serviço da ponte, com a conservação e manutenção da sua estrutura. Nesse contexto, o presente trabalho tem como objetivo avaliar o grau de deterioração da estrutura da ponte localizada no campus da Universidade Federal de Santa Maria. Para isso, será utilizada a metodologia do Grau de Deterioração da Estrutura (GDE/UnB), cuja versão mais recente foi proposta por Verly (2015). Para a aplicação da metodologia GDE/UnB, é necessário realizar uma inspeção visual da estrutura, onde avalia-se a ocorrência de manifestações patológicas e seu grau de intensidade. A partir dos resultados obtidos com o método, serão listadas as intervenções necessárias na ponte, em ordem de gravidade e urgência, buscando contribuir com o planejamento interno da universidade para a manutenção da sua infraestrutura.

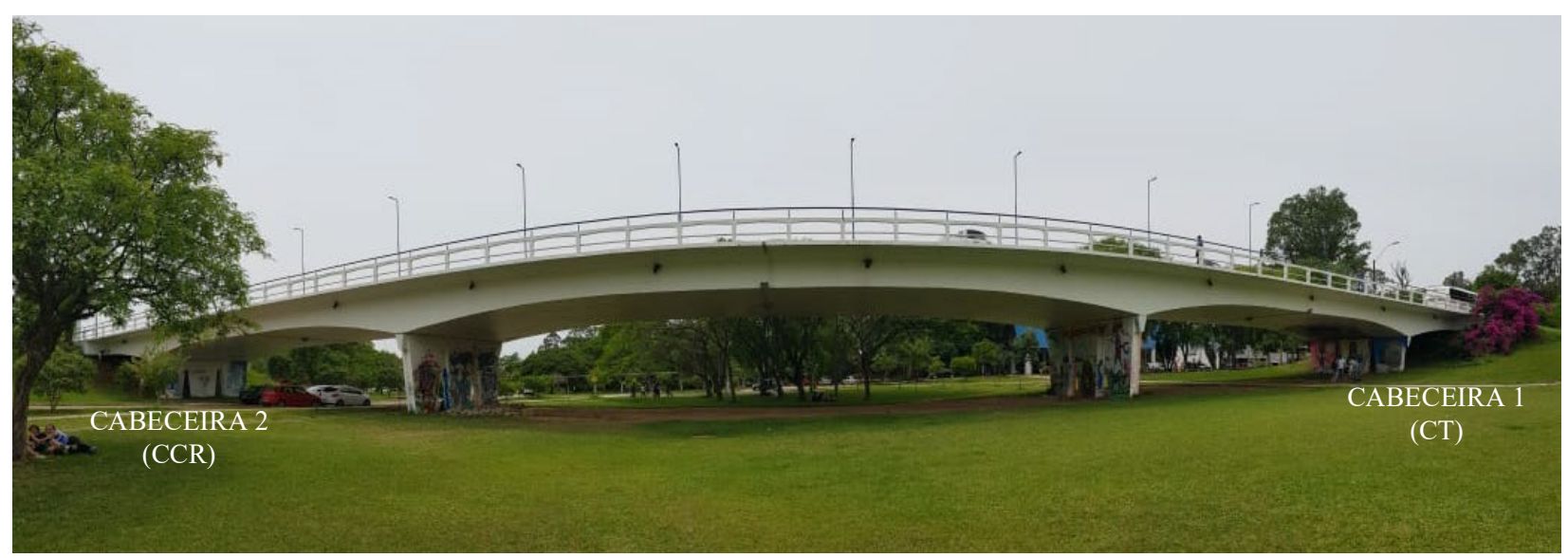

Figura 1. Vista geral da Ponte Marechal Cândido Mariano Rondon no campus da UFSM.

\section{METODOLOGIA GDE/UnB}

A metodologia do Grau de Deterioração da Estrutura (GDE/UnB) foi desenvolvida inicialmente por Castro (1994) para a avaliação de edificações e teve como base a metodologia de avaliação de OAEs proposta por Klein et al. (1991). A metodologia recebeu contribuições de Lopes (1998), Boldo (2002) e Fonseca (2007), e foi adaptada para a aplicação em OAEs por Euqueres (2011). A versão mais recente foi desenvolvida por Verly (2015) e será a metodologia adotada neste trabalho, pois é específica para a aplicação em OAEs.

A avaliação da OAE inicia com a divisão da estrutura em famílias de elementos típicos. Nessa etapa, os elementos estruturais são agrupados de acordo com suas características e função estrutural no conjunto. Elementos de uma mesma família são tratados da mesma forma pela metodologia, porém a divisão não é fixa e varia conforme o sistema estrutural da OAE analisada. Para estimar o grau de deterioração da estrutura, a metodologia GDE/UnB apresenta uma rotina de cálculo, que será detalhada nos itens a seguir.

\subsection{Fator de ponderação do dano $\left(F_{p}\right)$}

Durante a inspeção da $\mathrm{OAE}$, a metodologia GDE/UnB propõe a identificação de ocorrência dos seguintes tipos de danos nos elementos: carbonatação, cobrimento deficiente, contaminação por cloretos, corrosão de armaduras, desagregação, deslocamento por empuxo, desplacamento, desvios de geometria, eflorescência, falha de concretagem, fissuras, flechas excessivas, impermeabilização 
deficiente, infiltração de água, manchas, obstrução de juntas de dilatação, recalques e sinais de esmagamento do concreto. Para cada família típica de elementos estruturais, a metodologia $\mathrm{GDE} / \mathrm{UnB}$ fornece os fatores de ponderação do dano $\left(\mathrm{F}_{\mathrm{p}}\right)$ relativos a cada tipo de dano que pode ser observado nos elementos. Segundo Castro (1994), o objetivo do $F_{p}$ é quantificar a importância relativa de um determinado dano nas condições gerais de segurança, funcionalidade e estética dos elementos de uma determinada família. Isso permite que uma mesma manifestação patológica tenha fatores de ponderação distintos em famílias diferentes. Os valores de $F_{p}$ podem variar de 1 a 5 e são definidos antes da inspeção. A única exceção é no caso das fissuras, em que o valor de $\mathrm{F}_{\mathrm{p}}$ é definido no momento da inspeção de acordo com a natureza das fissuras observadas nos elementos.

\subsection{Fator de intensidade do dano $\left(F_{i}\right)$}

O fator de intensidade do dano tem como objetivo classificar a gravidade e a evolução isolada do dano em um determinado elemento, mas não diferencia o elemento estrutural no qual o dano se encontra. $\mathrm{O}$ valor de $\mathrm{F}_{\mathrm{i}}$ varia de 0 a 4 e cresce com a gravidade do estado em que o dano se encontra, como demonstra a Tabela 1. Para reduzir a subjetividade da avaliação, Verly (2015) apresenta uma relação de danos e recomendações de $\mathrm{F}_{\mathrm{i}}$, acompanhadas por fotos e desenhos no "Manual de aplicação da metodologia GDE/UnB a OAEs".

Tabela $1-$ Classificação do fator de intensidade do dano.
\begin{tabular}{|c|c|}
\hline Estado do elemento & $\mathbf{F}_{\mathbf{i}}$ \\
\hline Elemento sem lesões & 0 \\
\hline Elemento com lesões leves & 1 \\
\hline Elemento com lesões toleráveis & 2 \\
\hline Elementos com lesões graves & 3 \\
\hline Elemento em estado crítico & 4 \\
\hline
\end{tabular}

Fonte: Verly (2015).

\subsection{Grau do dano (D)}

A formulação do grau do dano foi introduzida por Castro (1994) e faz analogia com o modelo proposto por Tuutti (1982), que considera que o processo de deterioração de uma estrutura de concreto ocorre em duas fases distintas: iniciação e propagação. Para definir o grau do dano, Castro (1994) utilizou como variáveis o fator de intensidade do dano $\left(\mathrm{F}_{\mathrm{i}}\right)$ e o fator de ponderação do dano $\left(\mathrm{F}_{\mathrm{p}}\right)$, dando origem às Equações (1) e (2), que correspondem às fases de iniciação e propagação, respectivamente.

$$
\begin{array}{lrl}
D=0,8 \cdot F_{i} \cdot F_{p} & \text { para } & F_{i} \leq 2,0 \\
D=\left(12 \cdot F_{i}-28\right) \cdot F_{p} & \text { para } & F_{i}>2,0
\end{array}
$$

\subsection{Grau de deterioração do elemento $\left(G_{d e}\right)$}

Após a avaliação de cada dano isoladamente (D), deve-se analisar o elemento estrutural sob o efeito de todos os danos nele existentes, utilizando a Equação (3). Essa equação foi proposta por Lopes (1998) e tem como objetivo incluir os efeitos dos danos menores aos efeitos do maior dano encontrado no elemento ( $\left.D_{\text {máx }}\right)$. 


$$
G_{d e}=D_{m a ́ x} \cdot\left[1+\frac{\left(\sum_{i=1}^{n} D_{i}\right)-D_{m a ́ x}}{\sum_{i=1}^{n} D_{i}}\right]
$$

\subsection{Grau de deterioração de uma família de elementos $\left(G_{d f}\right)$}

O grau de deterioração de uma família de elementos $\left(\mathrm{G}_{\mathrm{df}}\right)$ tem como objetivo avaliar todos os elementos pertencentes a uma mesma família. Para isso, leva em consideração os valores de $\mathrm{G}_{\mathrm{de}}$ obtidos para cada elemento pertencente à família analisada, conforme a Equação (4).

$$
G_{d f}=G_{d e, m a ́ x} \cdot\left[1+\frac{\left(\sum_{i=1}^{m} G_{d e, i}\right)-G_{d e, m a ́ x}}{\sum_{i=1}^{m} G_{d e, i}}\right]
$$

\subsection{Fator de relevância estrutural $\left(F_{r}\right)$}

O fator de relevância estrutural tem como objetivo considerar a importância relativa de uma família de elementos no comportamento e no desempenho global da estrutura. Assim, o valor do $\mathrm{F}_{\mathrm{r}}$ depende do sistema estrutural em análise, sendo recomendados por Verly (2015) os valores da Tabela 2.

Tabela 2 - Fatores de relevância estrutural.

\begin{tabular}{|l|c|}
\hline \multicolumn{1}{|c|}{ Tipo de elemento } & Fr $_{\mathbf{r}}$ \\
\hline Barreiras de defensas, guarda-corpo, guarda-rodas, pista de rolamento & 1,0 \\
\hline Juntas de dilatação & 2,0 \\
\hline Transversinas, cortinas, alas & 3,0 \\
\hline Lajes, fundações, vigas secundárias, aparelhos de apoio & 4,0 \\
\hline Vigas principais e pilares & 5,0 \\
\hline
\end{tabular}

Fonte: Verly (2015).

\subsection{Grau de deterioração da estrutura $\left(G_{d}\right)$}

O grau de deterioração da estrutura como um todo é definido em função dos valores de $\mathrm{G}_{\mathrm{df}}$ obtidos para cada família de elementos da estrutura. A Equação (5) fornece o grau de deterioração da família majorado $(\mathrm{K})$, dado pelo produto do $\mathrm{G}_{\mathrm{d}, \mathrm{f}}$ e $\mathrm{F}_{\mathrm{r}}$.

Fonseca (2007) propôs a Equação (6) para determinar o grau de deterioração da estrutura $\left(\mathrm{G}_{\mathrm{d}}\right)$, na qual os valores de $G_{d f}$ são ponderados pelos respectivos fatores de relevância estrutural. Nesse caso, valores de $G_{\text {de }}$ menores que 15 devem ser desprezados no cálculo do $G_{\text {df. }}$ Verly (2015) modificou a equação anterior e propôs a Equação (7) para o cálculo do grau de deterioração da estrutura modificado $\left(\mathrm{G}_{\mathrm{d}, \bmod }{ }^{*}\right)$, no qual todos os valores de $\mathrm{G}_{\mathrm{de}}$ devem ser considerados no cálculo do $\mathrm{G}_{\mathrm{df}}$.

$$
\begin{aligned}
& K_{i}=G_{d f, i} \cdot F_{r, i} \\
& G_{d}=\frac{\sum_{i=1}^{k} K_{i}}{\sum_{i=1}^{k} F_{r, i}} \\
& G_{d, \bmod ^{*}}=\frac{K_{\text {máx }}}{7,07} \cdot \sqrt{1+\frac{\left(\sum_{i=1}^{k} K_{i}\right)-K_{m a ́ x}}{\sum_{i=1}^{k} K_{i}}}
\end{aligned}
$$


Após a definição do $\mathrm{G}_{\mathrm{d}}\left(\right.$ ou $\left.\mathrm{G}_{\mathrm{d} \text {,mod }}{ }^{*}\right)$ a estrutura pode ser classificada segundo a escala apresentada na Tabela 3, onde há a indicação do nível de deterioração e recomendações de ações a serem tomadas.

Tabela 3 - Classificação dos níveis de deterioração da estrutura e ações recomendadas.

\begin{tabular}{|c|c|c|}
\hline $\begin{array}{c}\text { Nível de } \\
\text { deterioração }\end{array}$ & $\mathbf{G}_{\mathbf{d}}$ & Ações recomendadas \\
\hline Baixo & $0-15$ & $\begin{array}{c}\text { Estado aceitável. } \\
\text { Manutenção preventiva. }\end{array}$ \\
\hline Médio & $15-50$ & $\begin{array}{l}\text { Definir prazo e natureza de nova inspeção. } \\
\text { Planejar intervenção em longo prazo (máx. } 2 \text { anos). }\end{array}$ \\
\hline Alto & $50-80$ & $\begin{array}{l}\text { Definir prazo para inspeção especializada. } \\
\text { Planejar intervenção em médio prazo (máx. } 1 \text { ano). }\end{array}$ \\
\hline Sofrível & $80-100$ & $\begin{array}{l}\text { Definir prazo para inspeção especializada rigorosa. } \\
\text { Planejar intervenção em curto prazo (máx. } 6 \text { meses). }\end{array}$ \\
\hline Crítico & $\geq 100$ & $\begin{array}{l}\text { Inspeção especializada imediata e medidas emergenciais (alívio } \\
\text { de cargas, escoramento, etc). Planejar intervenção imediata. }\end{array}$ \\
\hline
\end{tabular}

Fonte: Verly (2015).

\section{RESULTADOS E DISCUSSÃO}

Neste trabalho foi realizada uma inspeção visual, de forma que foram avaliados apenas os danos que puderam ser detectados visualmente na estrutura. Para isso, a OAE foi dividida nas seguintes famílias: pilares, travessas, laje (com seção caixão), cortinas, guarda-roda, guarda-corpo e pista de rolamento. Devido à presença de aterro nas cabeceiras da ponte não foi possível inspecionar a estrutura de aproximação, bem como os blocos de fundação, os aparelhos de apoio e o interior da seção caixão.

\subsection{Mesoestrutura}

A mesoestrutura da OAE é composta pelos pilares e vigas travessas. Existem quatro conjuntos de pilares, com três pilares por conjunto. As vigas travessas estão posicionadas acima dos pilares e fazem a distribuição do carregamento oriundo da superestrutura para os pilares do conjunto.

Os pilares, de forma geral, encontram-se em bom estado de conservação, recebendo pinturas recorrentes feitas pelos estudantes que utilizam o espaço para manifestações artísticas. Foram observados, no entanto, pequenos focos de desplacamento do concreto na base dos pilares (Figuras $2 \mathrm{a}$ e $2 \mathrm{~b}$ ), na maioria sem ocorrência de exposição das armaduras. As vigas travessas também se encontram em boas condições, tendo sido identificado apenas a ocorrência de fissuras em uma delas, e desplacamento do concreto com exposição da armadura em outra. Nesse caso, apesar da armadura estar sem o cobrimento de concreto, ela encontra-se coberta pela tinta utilizada na pintura (Figura 2c). 


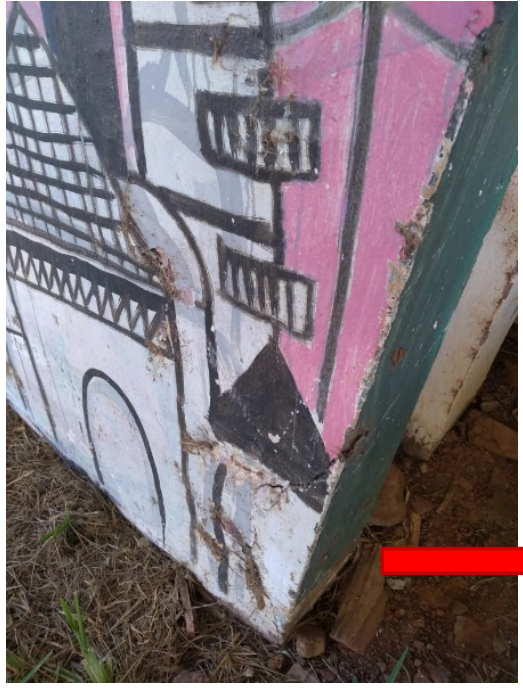

(a)

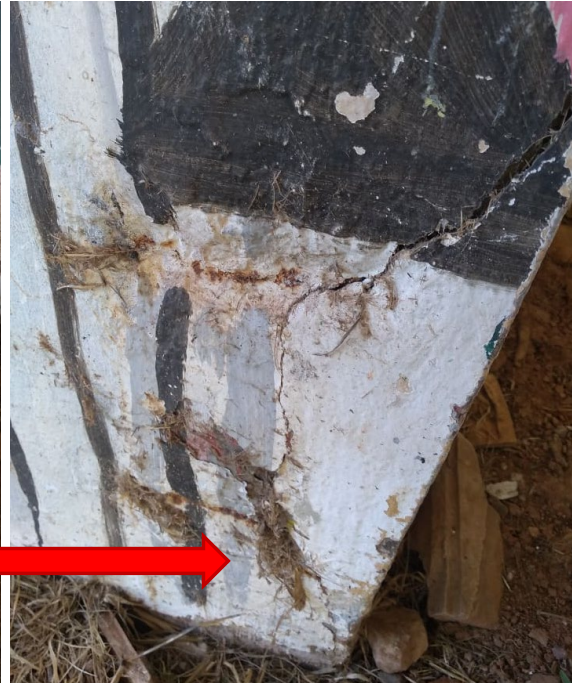

(b)

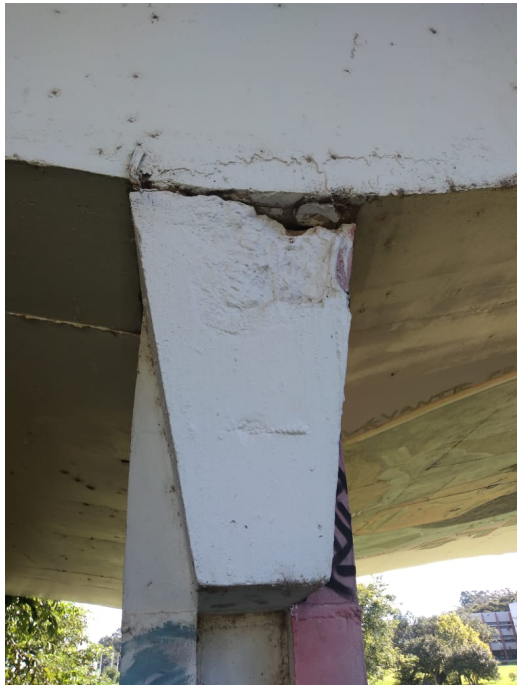

(c)

Figura 2. Desplacamento na base de pilar (a); detalhe do desplacamento (b); desplacamento com exposição da armadura em viga travessa (c).

\subsection{Superestrutura}

Na parte inferior da laje foram observados diversos pontos para drenagem da seção caixão (Figura 3), e buzinotes para a drenagem da pista de rolamento e passeio (Figura 5). Entretanto, vários pontos de drenagem parecem estar obstruídos, causando o surgimento de manchas (Figura 3a) e infiltrações (Figura 3b) na laje inferior da seção caixão.

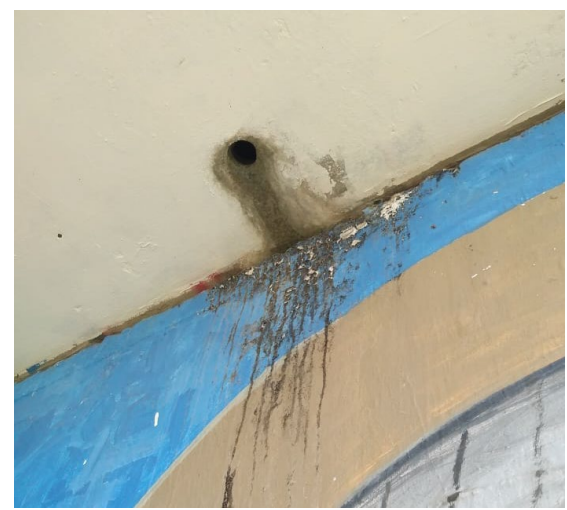

(a)

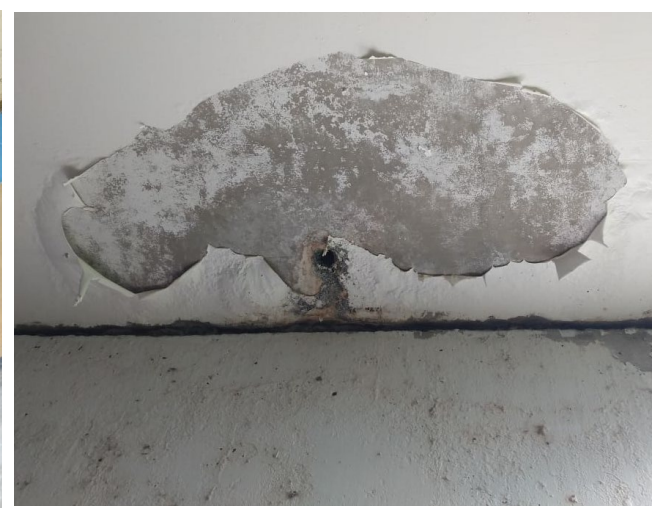

(b)

Figura 3. Vista da laje inferior da seção caixão com: manchas (a) e infiltração (b).

Nas lajes laterais, destinadas ao passeio de pedestres, foram observadas inúmeras fissuras (Figura 4) oriundas, possivelmente, da ausência de juntas de dilatação nos 108 metros de extensão da ponte. As lajes de passeio são engastadas na lateral da seção caixão, com a armadura principal disposta perpendicularmente ao eixo da ponte, de forma que apenas a armadura secundária da laje (de distribuição) é responsável por resistir aos esforços decorrentes da dilatação térmica da estrutura. As fissuras, portanto, teriam ocorrido pela deficiência da armadura disposta nesse sentido. Por outro lado, na seção caixão a armadura principal está posicionada longitudinalmente ao eixo da ponte e atua resistindo aos esforços oriundos da dilatação térmica, sendo este, provavelmente, o motivo pelo qual não foram localizadas fissuras com este padrão na laje inferior da seção caixão. Além disso, foi possível observar que as fissuras na laje de passeio do lado direito da ponte (Figura 4b) possuíam abertura muito superior às fissuras presentes na laje de passeio do lado esquerdo 
(Figura 4a). A deterioração das lajes do lado direito é agravada pelo sistema de drenagem da pista de rolamento, que direciona parte do volume de água aos buzinotes e o excedente para a laje de passeio, de onde a água escorre livremente pela lateral, provocando manchas, infiltrações e a corrosão das armaduras na face inferior da laje.

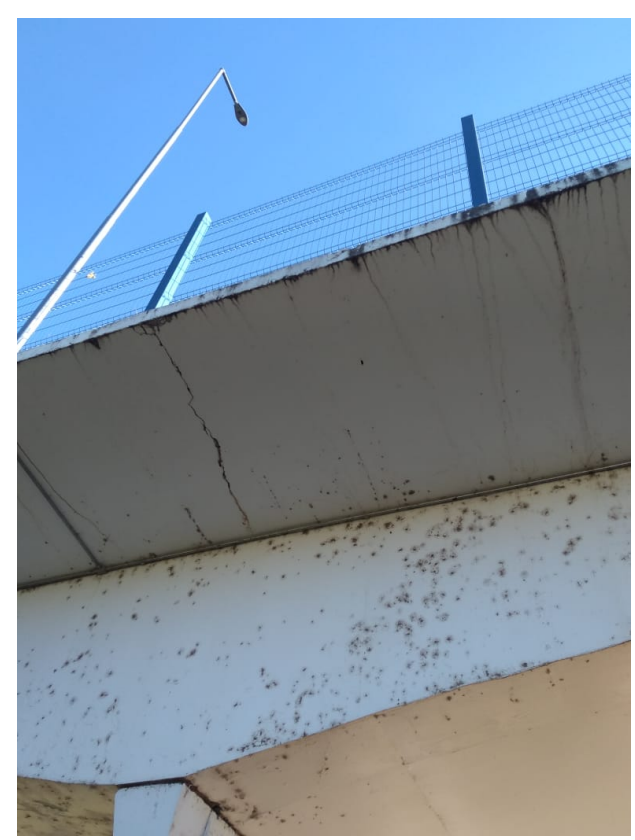

(a)

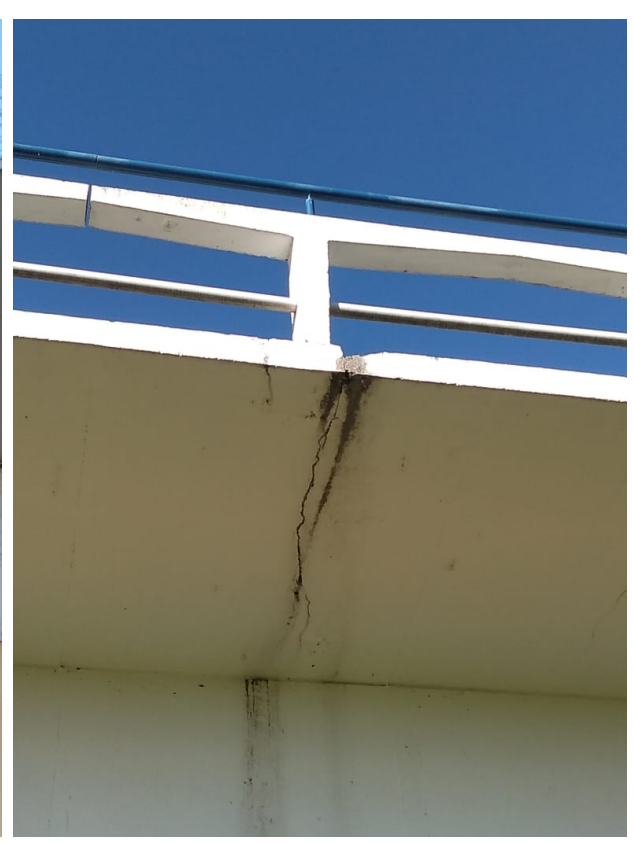

(b)

Figura 4. Fissuras transversais ao eixo da ponte localizadas nas lajes de passeio: (a) laje à esquerda da pista; (b) laje à direita da pista.

Apesar da metodologia GDE/UnB não incluir a avaliação da integridade dos aterros das cabeceiras da ponte, durante a inspeção foi possível observar que está ocorrendo a percolação de água pelo tabuleiro, com erosão significativa dos aterros nas cabeceiras (Figuras 5 e 6). A infiltração de água e presença de umidade nessa região está contribuindo para a deterioração das vigas cortinas, que já apresentam manchas e pontos de desplacamento do concreto com a exposição de armaduras, em processo avançado de corrosão.

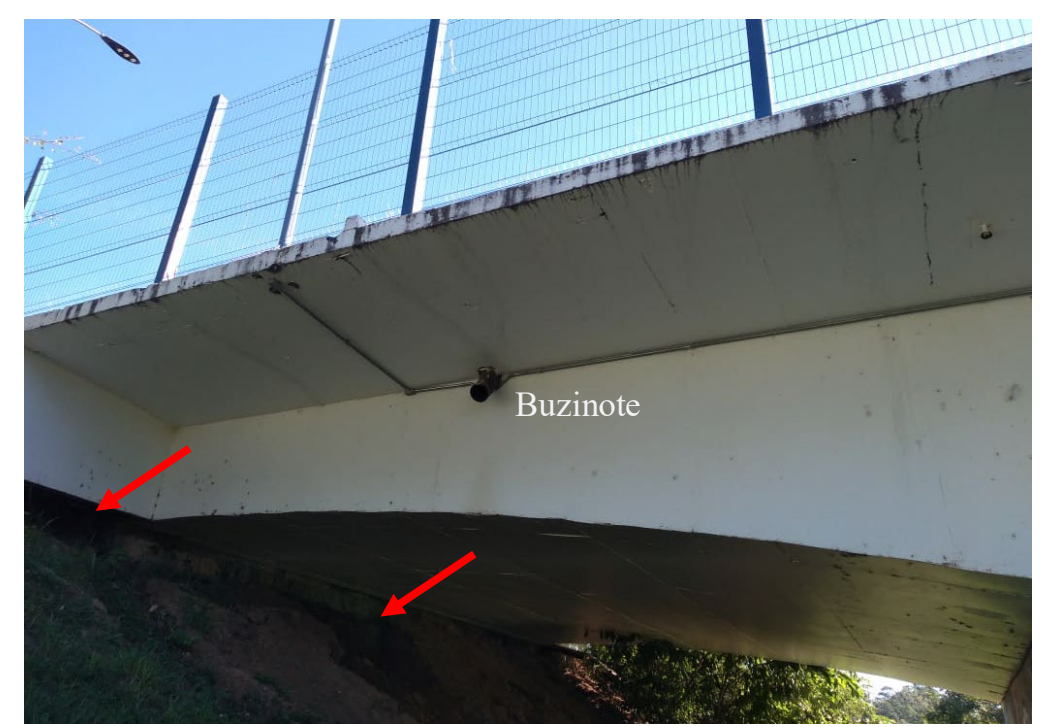

Figura 5. Vista lateral da ponte, com erosão no aterro da cabeceira devido à infiltração de água. 


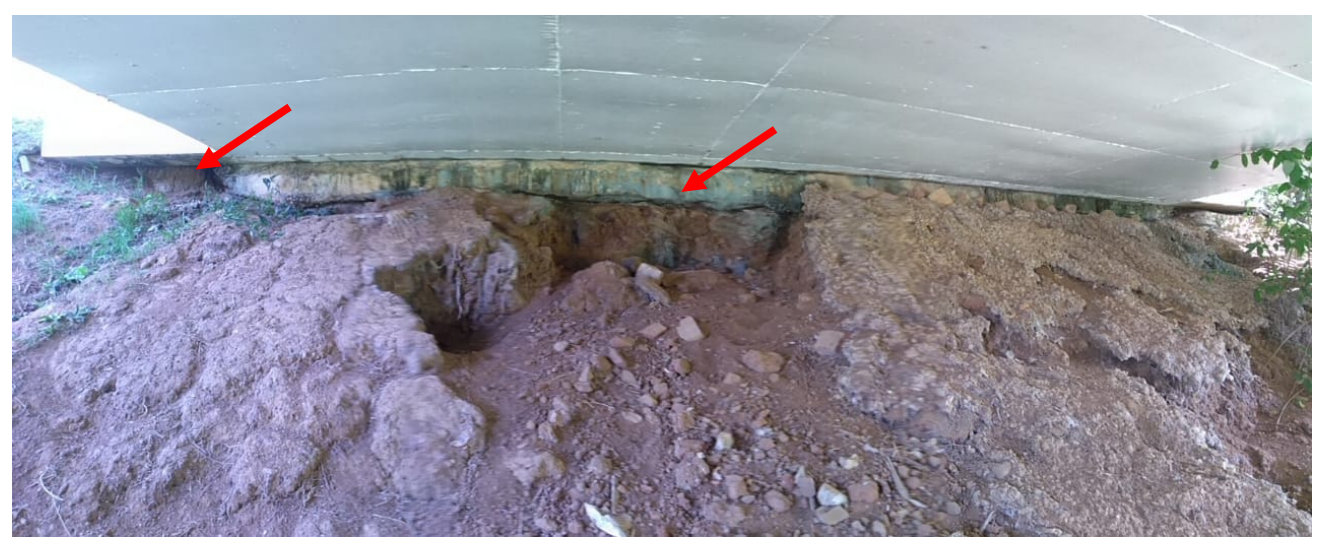

Figura 6. Vista inferior do tabuleiro, com erosão do aterro da cabeceira devido à infiltração de água.

Como consequência do processo de erosão dos aterros nas cabeceiras, na pista de rolamento é possível identificar uma descontinuidade no revestimento asfáltico (Figura 7a), bem como localizar o vão por onde ocorre a percolação da água, no encontro da superestrutura com o aterro de acesso (Figura 7b). Na Figura 7 (a) pode-se visualizar que há um desnível na pista pelo desnível observado no guarda-rodas. Além disso, na Figura 7 (b) fica evidente a grande dimensão do vão por onde está ocorrendo a percolação de água ao compara-lo com a espessura de uma lapiseira.

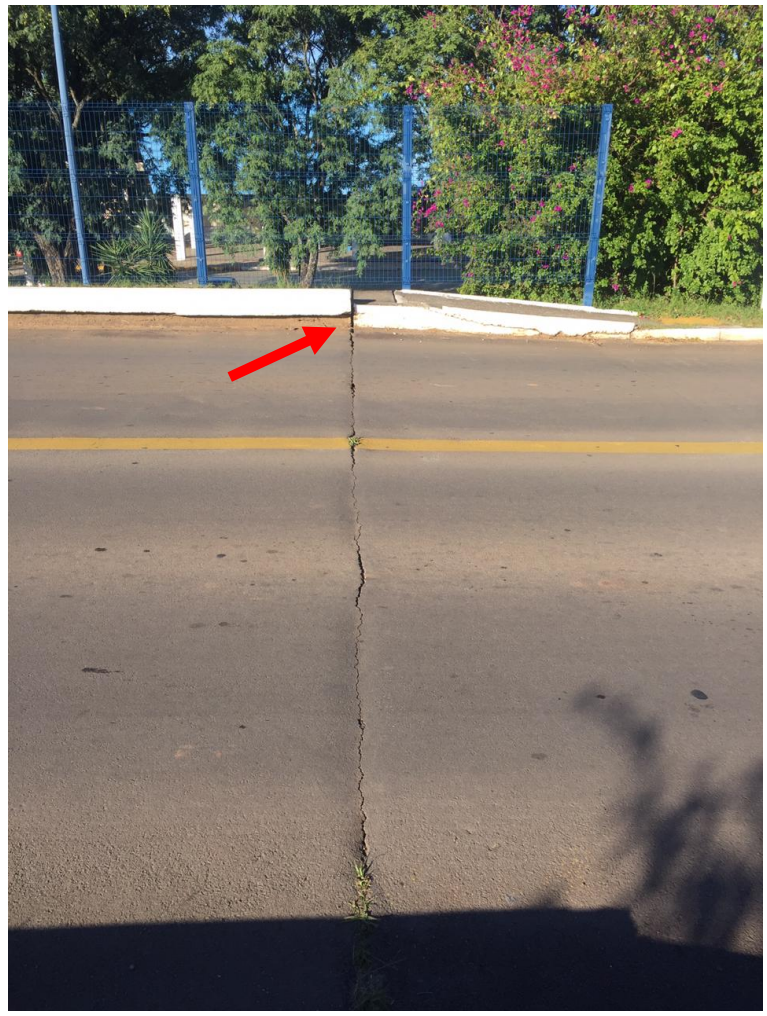

(a)

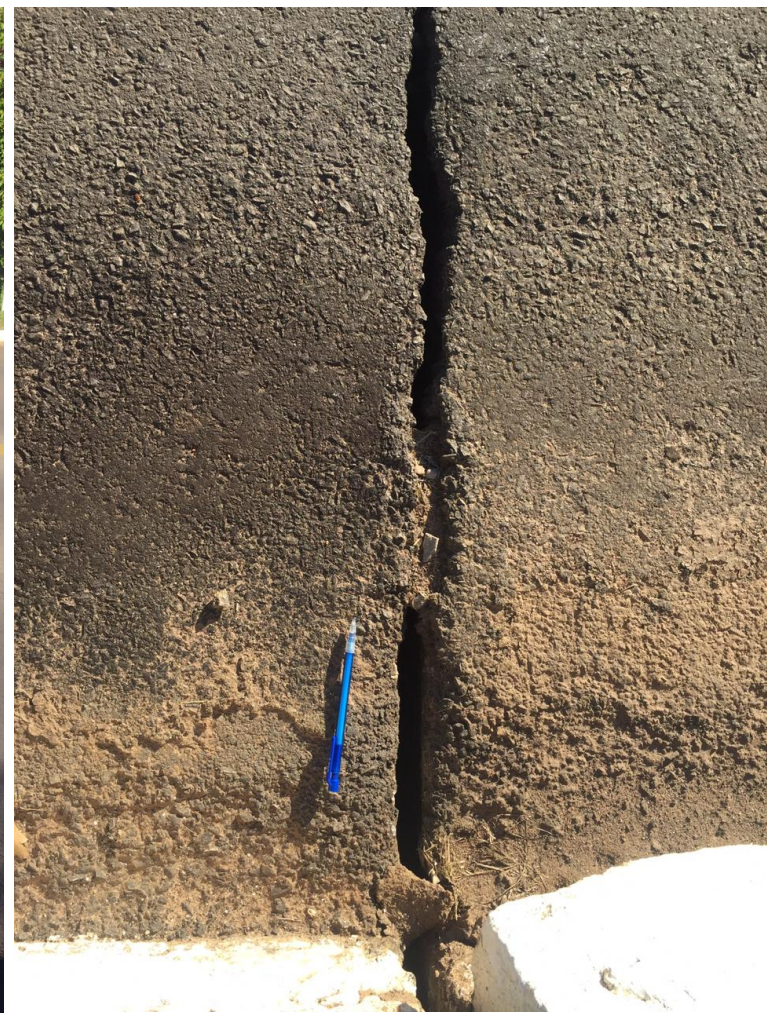

(b)

Figura 7. Cabeceira 2: descontinuidade na pista de rolamento e desnível no guarda-rodas (a); vão por onde ocorre a percolação de água (b).

Na cabeceira 1 a erosão do aterro provocou o surgimento de um degrau na pista, onde ocorre um forte impacto dos pneus dos veículos quando acessam a ponte, introduzindo esforços laterais adicionais na estrutura. Em medições realizadas in loco, foi constatado um desnível de $6 \mathrm{~cm}$ do 
lado esquerdo e de $8 \mathrm{~cm}$ do lado direito da cabeceira (Figura 8), sinalizando a redução de altura do aterro decorrente da erosão provocada pela percolação de água.

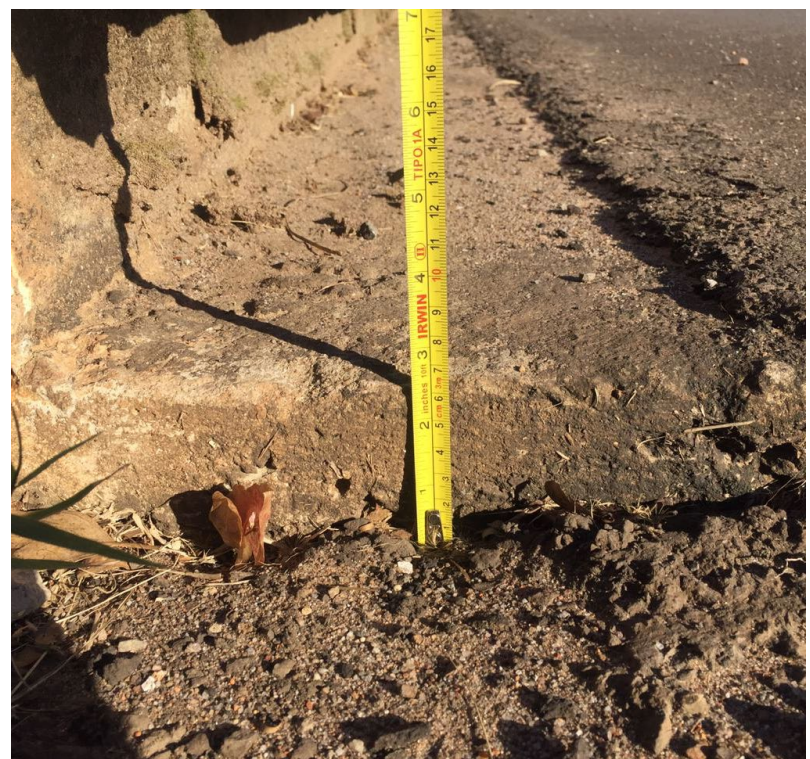

(a)

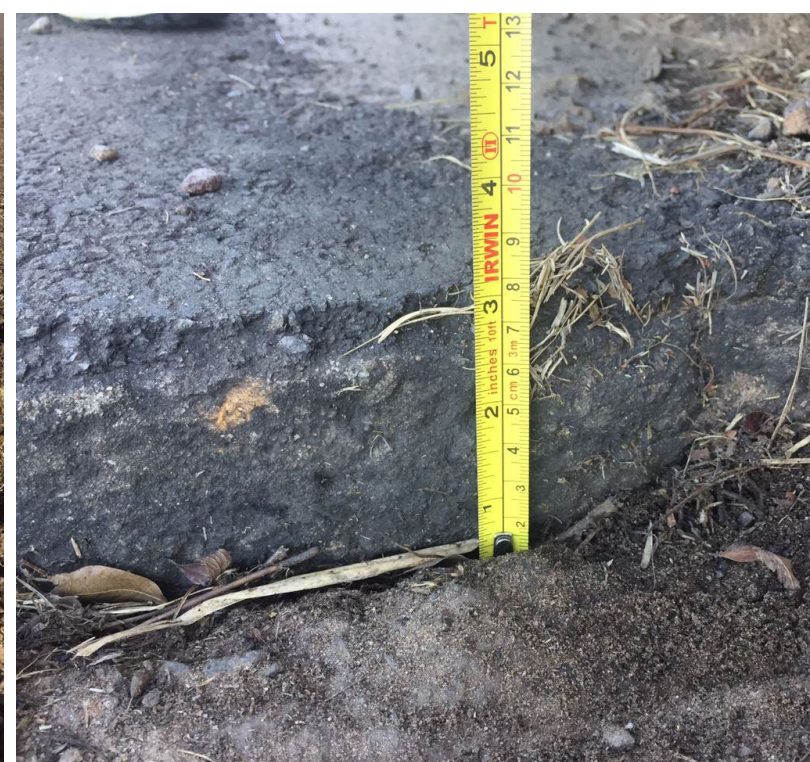

(b)

Figura 8. Cabeceira 1: desnível no acesso à ponte de $6 \mathrm{~cm}$ a esquerda (a) e $8 \mathrm{~cm}$ a direita (b).

Os guarda-rodas e o guarda-corpo, de forma geral, estavam em bom estado de conservação, com o guarda-rodas apresentando pequenas fissuras e o guarda-corpo com desagregação do concreto apenas em um ponto específico. Na pista de rolamento foi constatada uma descontinuidade grave (Figuras 7 e 8), um desgaste superficial leve do revestimento asfáltico e um desgaste tolerável da sinalização.

\subsection{Resultados da metodologia GDE/UnB}

De acordo com a metodologia GDE/UnB, a família de elementos que teve o pior resultado na avaliação do grau de deterioração foram as lajes, que apresentaram cobrimento deficiente, corrosão de armaduras, desplacamento do concreto, fissuras, infiltração de água e manchas. O resultado é consequência, principalmente, da situação em que se encontram as lajes de passeio, com inúmeras fissuras, manchas e com corrosão de armaduras, decorrentes da ausência de juntas de dilatação e agravadas pelo sistema de drenagem deficiente. A maioria dos vãos das lajes encontram-se com um grau de deterioração do elemento $\left(\mathrm{G}_{\mathrm{de}}\right)$ superior a 80 , indicando um nível de deterioração sofrível, para o qual a metodologia recomenda planejar uma intervenção em no máximo 6 meses, com a definição de um prazo para ocorrer uma inspeção especializada rigorosa.

Depois das lajes, o segundo pior resultado foi da família das vigas cortinas, devido a ocorrência de corrosão de armaduras, desplacamento do concreto, infiltração de água e manchas, que resultaram em um grau de deterioração do elemento $\left(\mathrm{G}_{\mathrm{de}}\right)$ superior a 60 nas duas cabeceiras, indicando um nível de deterioração alto. Nesse caso, o resultado negativo deve-se à percolação de água pelo tabuleiro, com erosão dos aterros das cabeceiras e deterioração das vigas cortinas. A metodologia GDE/UnB recomenda que uma inspeção especializada seja realizada e uma intervenção planejada para ocorrer em no máximo 1 ano.

Os pilares e as travessas obtiveram um nível de deterioração do elemento entre baixo e médio, para os quais a metodologia recomenda uma manutenção preventiva ou planejar uma intervenção em até 2 anos. Os guarda-rodas, guarda-corpo e pista de rolamento apresentaram um nível de deterioração baixo, sendo recomendada apenas uma manutenção preventiva. 
O grau de deterioração da estrutura, relativo ao estado geral de conservação da ponte, foi calculado de duas formas: usando a Equação (6) do $G_{d}$, de Fonseca (2007), e a Equação (7) do $G_{d, m o d}{ }^{*}$, de Verly (2015). A primeira equação chegou a um $\mathrm{G}_{\mathrm{d}}$ de 75,90 , indicando um nível de deterioração alto, enquanto a segunda equação obteve um $\mathrm{G}_{\mathrm{d} \text {,mod }}{ }^{*}$ de 102,95 , que indica um nível de deterioração crítico. Foi necessário calcular o grau de deterioração da família $\left(\mathrm{G}_{\mathrm{df}}\right)$ de forma independente para a aplicação nas equações do $\mathrm{G}_{\mathrm{d}}$ e $\mathrm{G}_{\mathrm{d} \text {,mod }}{ }^{*}$, pois na primeira deve-se considerar apenas os elementos com grau de deterioração $\left(\mathrm{G}_{\mathrm{de}}\right)$ maiores que 15 , enquanto a segunda considera todos os elementos. Os resultados são apresentados na Tabela 4.

Tabela 4 - Cálculo do Grau de Deterioração da Estrutura.

\begin{tabular}{|c|c|c|c|c|c|}
\hline Famílias de elementos & $\mathbf{F}_{\mathbf{r}}$ & $\mathbf{G}_{\mathbf{d f}}{ }^{(1)}$ & $\mathbf{G}_{\mathbf{d}}$ & $\mathbf{G}_{\mathbf{d f}}{ }^{(2)}$ & $\mathbf{G}_{\mathbf{d}, \bmod }{ }^{*}$ \\
\hline Pilares & 5,00 & 48,85 & \multirow{7}{*}{$\begin{array}{c}75,90 \\
\text { Alto }\end{array}$} & 51,73 & \multirow{7}{*}{$\begin{array}{l}102,95 \\
\text { Crítico }\end{array}$} \\
\hline Travessas & 5,00 & 36,00 & & 36,00 & \\
\hline Lajes & 4,00 & 144,31 & & 145,44 & \\
\hline Cortinas & 3,00 & 96,24 & & 96,24 & \\
\hline Guarda-rodas & 1,00 & 0,00 & & 10,29 & \\
\hline Guarda-corpo & 1,00 & 0,00 & & 4,80 & \\
\hline Pista de rolamento & 1,00 & 0,00 & & 14,77 & \\
\hline
\end{tabular}

(1) considera no cálculo do $\mathrm{G}_{\mathrm{df}}$ apenas os $\mathrm{G}_{\mathrm{de}}>15$.

(2) considera no cálculo do $\mathrm{G}_{\mathrm{df}}$ todos os $\mathrm{G}_{\mathrm{de}}$.

A partir dos resultados obtidos, como propostas de intervenções necessárias na ponte, em ordem de gravidade e urgência, sugere-se:

- melhorar o sistema de drenagem da pista, de forma a evitar que o volume excedente de água corra para as lajes de passeio, e proceder à instalação de pingadeiras nas lajes de passeio;

- recuperação e reforço das lajes de passeio, para que suportem os esforços oriundos da dilatação térmica da estrutura, evitando nova fissuração;

- selagem dos vãos entre o tabuleiro e o aterro da cabeceira, por onde está ocorrendo a percolação de água com erosão do aterro;

- recuperação das vigas cortinas;

- recomposição do aterro e nivelamento da pista de rolamento;

- manutenção nos drenos na seção caixão;

- manutenção preventiva nos pilares, travessas, guarda-rodas, guarda-corpo, no revestimento asfáltico e na sinalização da pista de rolamento.

\section{CONCLUSÕES}

Neste trabalho realizou-se a avaliação do grau de deterioração da estrutura da ponte localizada dentro do campus da Universidade Federal de Santa Maria, no RS. A partir da inspeção visual da estrutura, fez-se o levantamento das manifestações patológicas (danos) presentes em todos os elementos estruturais que compõem a $\mathrm{OAE}$ e fatores de intensidade foram atribuídos para cada dano, possibilitando a determinação do grau de deterioração do elemento $\left(G_{\text {de }}\right)$, da família de elementos $\left(\mathrm{G}_{\mathrm{df}}\right)$ e da estrutura como um todo $\left(\mathrm{G}_{\mathrm{d}}\right.$ ou $\left.\mathrm{G}_{\mathrm{d} \text {,mod }}{ }^{*}\right)$.

Duas equações foram utilizadas para calcular o grau de deterioração da estrutura e apresentaram resultados muito distintos. A equação proposta por Fonseca (2007) para o cálculo do $\mathrm{G}_{\mathrm{d}}$ resultou em um nível de deterioração alto para a ponte, o qual condiz mais com a realidade observada in loco. A equação proposta por Verly (2015) para o cálculo do $\mathrm{G}_{\mathrm{d} \text {,mod }}{ }^{*}$ resultou em um nível de deterioração crítico, entretanto, na inspeção in loco fica evidente que o estado de conservação geral 
da estrutura é bom, sendo crítico o estado de deterioração apenas das lajes de passeio e das cabeceiras da ponte. Assim, considerando o grau de deterioração da estrutura $\left(\mathrm{G}_{\mathrm{d}}\right)$ de 75,90 , a metodologia GDE/UnB recomenda que uma inspeção especializada seja realizada, e uma intervenção deve ser planejada para ocorrer em no máximo um ano.

A metodologia GDE/UnB mostrou-se eficaz para definir a ordem de prioridade de intervenções na estrutura da ponte. Destaca-se que a avaliação apresentada neste trabalho foi fruto apenas da inspeção visual da estrutura; ainda assim, com a metodologia GDE/UnB foi possível identificar e localizar as manifestações patológicas mais graves e em estado mais avançado de deterioração.

\section{REFERÊNCIAS}

Boldo, P. (2002), "Avaliação quantitativa de estruturas de concreto armado de edificações no âmbito do Exército Brasileiro", Dissertação (Mestrado), Departamento de Engenharia Civil e Ambiental, Universidade de Brasília, Brasília, 295 p.

Castro, E. K. (1994), "Desenvolvimento de metodologia para manutenção de estruturas de concreto armado", Dissertação (Mestrado), Departamento de Engenharia Civil e Ambiental, Universidade de Brasília, Brasília, 185 p.

Euqueres, P. (2011), "Metodologia de inspeção de pontes de concreto armado", Dissertação (Mestrado), Escola de Engenharia Civil, Universidade Federal de Goiás, Goiânia, 168 p.

Fonseca, R. P. (2007), "A estrutura do Instituto Central de Ciências: Aspectos históricos, científicos e tecnológicos de projeto, execução, intervenções e propostas de manutenção", Dissertação (Mestrado), Departamento de Engenharia Civil e Ambiental, Universidade de Brasília, Brasília, 213 p.

Helene, P. R. L. (2003), "Manual de reparo, proteção e reforço de estruturas de concreto". São Paulo, Red Rehabilitar.

Klein, D. L.; Gastal, F. P. S. L.; Campagnolo, J. L.; Silva Filho, L. C. P. (1991), "Critérios adotados na vistoria e avaliação de obras de arte" in: XXV Jornadas Sul-Americanas de Engenharia Estrutural, Porto Alegre: UFRGS, pp. 185-197.

Lopes, B. A. R. (1998), "Sistema de manutenção predial para grandes estoques de edifícios: estudo para inclusão do componente "estrutura de concreto"", Dissertação (Mestrado), Departamento de Engenharia Civil e Ambiental, Universidade de Brasília, Brasília, 308 p.

Tuutti, K. (1982), "Corrosion steel in concrete", Swedish Cement and Concrete, Research Institute, Stockholm.

Verly, R. C. (2015), "Avaliação de metodologias de inspeção como instrumento de priorização de intervenções em obras de arte especiais", Dissertação (Mestrado), Departamento de Engenharia Civil e Ambiental, Universidade de Brasília, Brasília, 178 p. 